\title{
The effects of bone metabolism in different methylprednisolone pulse treatments for Graves' ophthalmopathy
}

\author{
YONG-XIN HU ${ }^{1,2}$, REN-DONG ZHENG ${ }^{2}$, YAO-FU FAN ${ }^{2}$, LI SUN $^{2}, \mathrm{XIN} \mathrm{HU}^{2}$ and CHAO LIU ${ }^{2}$ \\ ${ }^{1}$ Laboratory of Endocrinology and Metabolism, The Third Clinical Medical College, Nanjing University of Chinese Medicine, \\ Nanjing, Jiangsu 210013; ${ }^{2}$ Department of Endocrinology, Affiliated Hospital of Integrated Traditional Chinese \\ and Western Medicine, Nanjing University of Chinese Medicine, Nanjing, Jiangsu 210028, P.R. China
}

Received October 10, 2018; Accepted May 2, 2019

DOI: $10.3892 /$ etm.2019.8179

\begin{abstract}
The aim of the present study was to analyze the effects of methylprednisolone pulse therapy (MPPT) courses on bone metabolism in patients with Graves' ophthalmopathy (GO). A retrospective analysis of 45 patients with moderate-to-severe active GO who received 1 or 2 courses of MPPT was performed. Of these, 16 patients underwent 2 courses of treatment. Bone metabolic markers and the density of the lumbar spine (L1-4), femoral neck and total hip were measured using a dual-energy X-ray bone density instrument, and the differences in bone metabolism prior to and after treatment were determined for each group and compared. The results indicated that serum I collagen $\mathrm{N}$-terminal peptide (P1NP) and serum $\beta$-collagen crosslinked C-terminal peptide (CTX) were markedly decreased after the first pulse of treatment. In those patients who received a second course of MPPT, CTX levels were significantly decreased, but P1NP was not significantly different from the baseline value. CTX and P1NP levels remained unchanged between the first and second course of MPPT; similarly, there were no changes from baseline in $25(\mathrm{OH})$ vitamin D3 and bone mineral density after the first and second course of MPPT. However, the level of $25(\mathrm{OH})$ vitamin D3 was significantly elevated after the second course compared with the first course. In conclusion, the side effects of MPPT on bone metabolism were marginal and a second course of MPPT did not worsen bone metabolism. These MPPT regimens may therefore be considered to be a safe and effective treatment option for patients with moderate-to-severe active $\mathrm{GO}$.
\end{abstract}

Correspondence to: Professor Chao Liu, Department of Endocrinology, Affiliated Hospital of Integrated Traditional Chinese and Western Medicine, Nanjing University of Chinese Medicine, 100 Hongshan Road, Nanjing, Jiangsu 210028, P.R. China

E-mail: profliuchao@163.com

Key words: Graves' ophthalmopathy, methylprednisolone pulse therapy, bone metabolism

\section{Introduction}

Graves' ophthalmopathy (GO), also called thyroid-associated ophthalmopathy, is an ocular disease that may potentially lead to loss of sight. Approximately $3-5 \%$ of patients with GO have severe disease with intense pain, inflammation and sight-threatening corneal ulceration or compressive optic neuropathy (1). Although novel evidence on associated immune mechanisms has provided a basis to explore other drug classes, corticosteroids are currently the mainstay of treatment (2). Different routes of administration and dosages have been used and various studies have attempted to identify the optimal regimen (3). Meta-analyses of randomized clinical trials clearly demonstrate that intravenous methylprednisolone pulses (IVMP) have greater effcacy than oral prednisone and fewer side-effects $(3,4)$. Consequently, guidelines recommend IVMP for active, moderate-to-severe GO, preferably $500 \mathrm{mg}$ weekly for 6 weeks followed by $250 \mathrm{mg}$ weekly for another 6 weeks (cumulative dose, 4.5 g) (5). Thus, 12-week high-dose methylprednisolone pulse therapy (MPPT) is now internationally recognized as a first-line treatment program for individuals with moderate-to-severe GO (6). However, glucocorticoids (GC) exert multiple side effects, the most notable of which is its negative effect on bone metabolism, which depends on the total dosage of GC administered (7). Hyperthyroidism concomitant with or ahead of GO may itself have adverse effects on bone tissue, and the ideal treatment should not further aggravate bone resorption. The curative effect of MPPT treatment has been clinically recognized and it may improve patient photophobia, lacrimation, pain and appearance (2); but its effect on bone metabolism has remained to be determined. The present retrospective analysis aimed to further clarify the effect of this treatment regimen on overall bone metabolism.

\section{Patients and methods}

General information. The present study was a retrospective analysis of real-world medical records. The data of all consecutive patients diagnosed with moderate-to-severe and active GO, who had completed the full course of IVMP therapy at the Endocrine and Diabetes Center of the Affiliated Hospital of Integrated Traditional Chinese and Western Medicine 
(Nanjing, China) from October 2014 to September 2017, were collected. All Patients were diagnosed with GO based on the Bartley criteria (8) and the European Group on Graves' Orbitopathy (EUGOGO) criteria (9). The clinical activity score (CAS) scoring standard was used, where a total score of 7 and a CAS $\geq 3$ indicates activity (10). The present study included patients with medium and severe active GO; i.e., with a CAS score of $>3$ points. To eliminate the effect of hyperthyroidism on bone metabolism, all patients required basic control of their hyperthyroidism, with Serum free triiodothyronine (FT3), free thyroxine (FT4) within the normal range. Thyroid-stimulating hormone (TSH) levels were normal or below the normal range. Patients with serious hyperthyroidism or refractory hyperthyroidism were excluded. Patients were excluded if they had the following co-morbidities: Hyperparathyroidism, diabetes mellitus, rheumatoid arthritis, ankylosing spondylitis, malignant tumor bone metastasis, severe liver or kidney dysfunction, or current or past treatment with GCs, anti-epileptic drugs, estrogen, heparin, progesterone, or other drugs that affect bone metabolism. Finally, the data of 45 patients (19 males and 26 females) with a disease course ranging between 2 months and 3 years were retrospectively collected and analyzed. The average year of the patients was $42.5 \pm 13.24$ years (range, 18-62 years). All patients provided written informed consent regarding the use of their clinical data for scientific research when they just entered the hospital.

Methods. Blood (10 ml) was collected from all patients and subjected to laboratory analyses on the second day after admission. The Roche Cobas 8000 Biochemical Analyzer (Roche Diagnostics) was used to determine serum alkaline phosphatase (ALP) and blood calcium (Ca). TSH was analyzed using a Siemens Centaur XP chemiluminescence detector (Siemens AG) and serum I collagen $\mathrm{N}$-terminal peptide (P1NP), $\beta$-collagen crosslinked C-terminal peptide (CTX) and 25-hydroxyvitamin D3 [25(OH)D3] were evaluated with a Roche e601 electrochemiluminescence detector (Roche Diagnostics). The BMD of lumbar vertebrae L1-4 and the left femoral neck were measured using a DEXA instrument from the GE-Lunar Prodigy Advance (GE Healthcare). The bone markers and bone mineral density (BMD) were reviewed prior to and after treatment in the single-pulse group for 3 months; and for the 2-pulse group, the bone metabolic index was again reviewed for 3 months, after the second pulse was completed. The average coefficient of variation for all anatomical sites measured was $0.92 \pm 0.42 \%$ and the BMD coefficient was $0.32 \%$.

During the course of routine evaluation, patients were divided into groups that were administered the following MPPT protocols: Methylprednisolone, $0.5 \mathrm{~g}$ once per week by intravenous drip for 6 consecutive weeks; then methylprednisolone, $0.25 \mathrm{~g}$ by intravenous drip once per week for 6 consecutive weeks, for a total of 12 weeks. All subjects received treatment with omeprazole $(40 \mathrm{mg}$ intravenous injection), potassium chloride ( $20 \mathrm{ml}$ orally prior to intravenous methylprednisolone) and supplementation with calcium (600 mg qd) and alfacalcidol (0.25 $\mu \mathrm{g} \mathrm{qd})$ until the end of the pulse. The treatment lasted for 3 months and was followed by a 3-month interval. After 6 months, based on the status of the first pulse treatment, it was decided whether to pursue the second course of pulse therapy using the same protocol as above. Treatment of hyperthyroidism was dependent on whether metronidazole or oral administration of propofol was used, and the FT3 and FT4 treatment was adjusted accordingly to allow the patient to remain within the normal range.

A total of 16 individuals were subjected to a second course of pulse therapy, which was consistent with the first course of treatment and adjuvant medication. The dosage for the pulse therapy in the second course was consistent with the first course and no other immunosuppressants were added.

Statistical analyses. SPSS 16.0 statistical software (SPSS, Inc.) was used for statistical analyses. Counting data were analyzed using a Chi-square test. The measurement data (expressed as the mean \pm standard deviation), Mean comparisons between the two groups were performed using a t test for normally distributed data. Non-normally distributed data were analyzed using a rank sum test; A group design $t$ test was used to compare two groups, and a paired t-test was used before and after treatment. A One-way ANOVA was used to compare the data among multiple groups, and pairwise comparison between groups was performed using a LSD test. $\mathrm{P}<0.05$ was considered to indicate a statistically significant difference.

\section{Results}

Comparison of baseline data. The baseline data, including TSH, were compared between sex and different age groups ( $<50$ or $\geq 50$ years). There were no statistically significant differences between the groups $(\mathrm{P}>0.05$, Table I).

Effect of single-pulse therapy on serum Ca, PTH, PINP and $C T X$ levels, and BMD. Following single-pulse therapy, serum $\mathrm{Ca}, \mathrm{PTH}, \mathrm{P} 1 \mathrm{NP}, \mathrm{CTX}$ levels, and BMD were compared with the baseline. The results indicated that serum $\mathrm{Ca}, \mathrm{PTH}$ and P1NP levels exhibited no differences after one pulse, however, CTX level after therapy in the single-pulse treatment group was lower than levels prior to treatment $(\mathrm{P}<0.05$; Fig. 1), but there was no statistically significant difference in BMD prior to vs. after treatment (Fig. 2).

Comparison of 25(OH)D3, P1NP and CTX levels between different age groups and sex prior to and after the first treatment. The CTX levels in individuals aged $\geq 50$ years were significantly lower after treatment $(\mathrm{P}<0.05)$, while other biochemical markers were similar between the 2 groups after treatment (Table II). While there were no significant differences in the indices among females upon treatment (Fig. 3), CTX was significantly decreased in males after treatment (Fig. 4).

The second pulse of therapy does not significantly affect PINP and CTX levels. A total of 16 patients were subjected to a second course of therapy. Compared with the base line level, CTX levels were reduced after the first therapy and after the second therapy, however there were no significant differences between CTX levels after the first and second therapy. In contrast, P1NP level exhibited no significant changes between baseline levels and after the second therapy. A statistical difference was only exhibited between baseline and first therapy, Compared to the initial levels of $25(\mathrm{OH}) \mathrm{D} 3$, no significant differences 
Table I. Comparison of baseline data prior to therapy.

\begin{tabular}{|c|c|c|c|c|c|}
\hline \multirow[b]{2}{*}{ Parameter } & \multirow[b]{2}{*}{ Normal ranges } & \multicolumn{2}{|c|}{ Sex } & \multicolumn{2}{|c|}{ Age (years) } \\
\hline & & Male $(n=19)$ & Female $(n=26)$ & $\geq 50(n=20)$ & $<50(\mathrm{n}=25)$ \\
\hline TSH (UIU/ml) & $0.27-4.2$ & $2.78 \pm 4.50$ & $1.24 \pm 2.01$ & $1.78 \pm 2.25$ & $1.98 \pm 4.05$ \\
\hline $\mathrm{Ca}(\mathrm{mmol} / \mathrm{l})$ & $2.15-2.55$ & $2.26 \pm 0.12$ & $2.25 \pm 0.09$ & $2.24 \pm 0.12$ & $2.27 \pm 0.09$ \\
\hline $\operatorname{ALP}(\mathrm{mmol} / \mathrm{l})$ & $50-135$ & $98.63 \pm 31.48$ & $95.96 \pm 49.09$ & $91.80 \pm 24.86$ & $101.32 \pm 52.21$ \\
\hline PTH (pg/ml) & $15-65$ & $42.90 \pm 13.52$ & $49.13 \pm 18.16$ & $48.54 \pm 16.73$ & $44.86 \pm 16.45$ \\
\hline 25(OH)D3 (ng/ml) & $>30$ & $18.41 \pm 5.73$ & $14.55 \pm 6.85$ & $16.49 \pm 7.00$ & $15.93 \pm 6.42$ \\
\hline P1NP (ng/ml) & $9.06-76.24$ & $79.36 \pm 44.40$ & $115.42 \pm 93.62$ & $81.48 \pm 36.62$ & $115.16 \pm 98.06$ \\
\hline CTX (ng/dl) & $0-70.4$ & $81.60 \pm 35.15$ & $87.74 \pm 33.59$ & $83.49 \pm 22.67$ & $86.48 \pm 41.33$ \\
\hline \multicolumn{6}{|l|}{$\mathrm{BMD}\left(\mathrm{g} / \mathrm{cm}^{2}\right)$} \\
\hline L1-4 & & $1.15 \pm 0.12$ & $1.10 \pm 0.14$ & $1.08 \pm 0.12$ & $1.15 \pm 0.14$ \\
\hline Femoral neck & & $0.91 \pm 0.11$ & $0.92 \pm 0.13$ & $0.88 \pm 0.11$ & $0.94 \pm 0.12$ \\
\hline Total hip & & $0.96 \pm 0.11$ & $0.93 \pm 0.13$ & $0.93 \pm 0.12$ & $0.96 \pm 0.12$ \\
\hline
\end{tabular}

Values are expressed as the mean \pm standard deviation. BMD, bone mineral density; ALP, alkaline phosphatase; PTH, parathyroid hormone; 25(OH)D3, 25-hydroxyvitamin D3; P1NP, I collagen N-terminal peptide; CTX, $\beta$-collagen crosslinked C-terminal peptide.

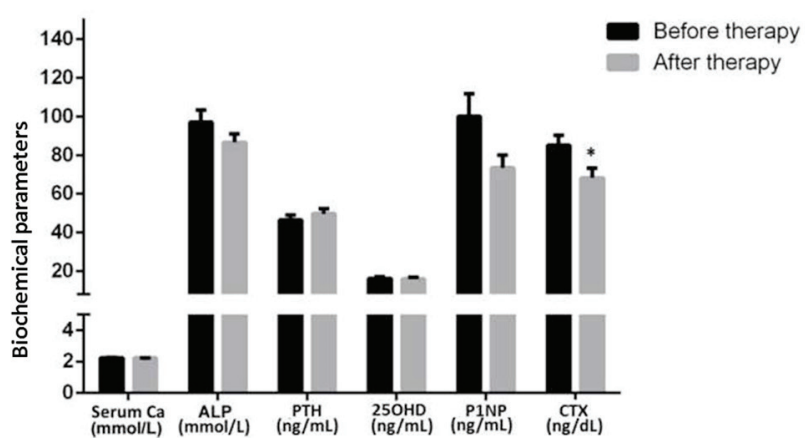

Figure 1. Comparison of serum $\mathrm{Ca}^{2+}, 25(\mathrm{OH}) \mathrm{D} 3, \mathrm{ALP}, \mathrm{PTH}, \mathrm{P} 1 \mathrm{NP}$ and CTX levels in the 2 groups after single-pulse treatment. After single-pulse treatment, ALP, P1NP and CTX levels of patients were lower compared with those prior to treatment. However, only CTX levels were significantly decreased after treatment. "P<0.05. ALP, alkaline phosphatase; PTH, parathyroid hormone; 25(OH)D3, 25-hydroxyvitamin D3; P1NP, I collagen N-terminal peptide; CTX, $\beta$-collagen crosslinked $\mathrm{C}$-terminal peptide.

were observed after either the first or second course of MPPT. However, compared with that after the first course, the level of $25(\mathrm{OH}) \mathrm{D} 3$ was significantly elevated after the second course of MPPT (Fig. 5). There were no significant differences in the BMD in different anatomical sites after treatment (Fig. 6).

\section{Discussion}

GO is an autoimmune disease closely associated with Graves' disease, that may or may not be accompanied by hyperthyroidism. Due to the inflammation and fibrosis of the extraocular muscles and orbital connective tissues, certain signs/symptoms, including increased volume of orbital contents, eyeball protrusion, eyelid retraction, bulbar conjunctival edema, eye movement disorder, diplopia and optic nerve oppression may appear. Approximately 3-5\% of GO patients progress to severe eye impairment (11). At present, the treatment options for moderate and severe GO are GC hormone therapy and orbital

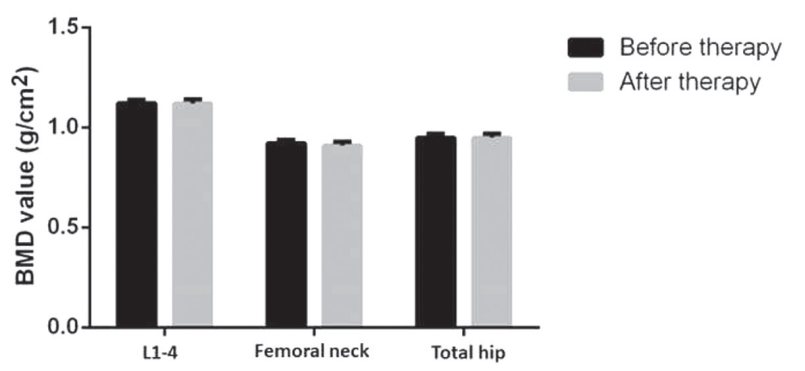

Figure 2. Comparison of BMD $\left(\mathrm{g} / \mathrm{cm}^{2}\right)$ at different anatomic sites of the 2 groups of patients. There was no significant difference in BMD between females prior to and after one course treatment. BMD, bone mineral density.

radiotherapy. GC reduce the adhesions of polysaccharide deposition, have anti-inflammatory and immune inhibitory effects and produce a more beneficial effect compared with GO, such as improving periorbital edema, congestion, altered ocular motility and diplopia, therefore, they are currently recognized as the most effective treatment.

The adverse effects of GC on bone, however, are noteworthy. Most patients receiving GC have bone loss leading to osteoporosis, which then increases the risk of fracture shortly after the initiation of treatment, particularly of the spinal vertebrae (12). Certain patients also develop bone necrosis. At present, oral GC for 3-6 months may lead to rapid bone loss and increase the risk of fracture, but the effect of intravenous drug use on bone metabolism has remained elusive. The primary direct effects of GC on bone formation are augmentation of peroxisome proliferator-activated receptor- $\gamma 2$ receptors and modulation of the $\mathrm{Wnt} / \beta$-catenin-signaling pathway, resulting in a decrease in the number of osteoblast cells and an increase in osteoblast and bone cell apoptosis. GC also directly stimulate bone resorption, although this effect decreases over time, possibly due to a decrease in the number of osteoblasts and bone cells (13). 
Table II. Comparison of 25(OH)D3, P1NP and CTX between individuals $\geq 50$ or $<50$ years of age prior to vs. after one course of treatment.

\begin{tabular}{|c|c|c|c|c|c|c|c|}
\hline $\begin{array}{l}\text { Age } \\
\text { (years)/time-point }\end{array}$ & $\mathrm{n}$ & $\begin{array}{c}\text { serum } \mathrm{Ca} \\
\mathrm{mmol} / \mathrm{l}\end{array}$ & $\begin{array}{c}\text { ALP } \\
\mathrm{mmol} / \mathrm{l}\end{array}$ & $\begin{array}{l}\mathrm{PTH} \\
\mathrm{ng} / \mathrm{ml}\end{array}$ & $\begin{array}{c}\text { 25(OH)D3 } \\
(\mathrm{ng} / \mathrm{ml})\end{array}$ & $\begin{array}{l}\text { P1NP } \\
(\mathrm{ng} / \mathrm{ml})\end{array}$ & $\begin{array}{l}\text { CTX } \\
(\mathrm{ng} / \mathrm{dl})\end{array}$ \\
\hline \multicolumn{8}{|l|}{$\geq 50$} \\
\hline Prior to & 20 & $2.24 \pm 0.12$ & $91.80 \pm 24.86$ & $48.54 \pm 16.73$ & $16.49 \pm 7.00$ & $81.48 \pm 36.63$ & $83.49 \pm 22.67^{\mathrm{a}}$ \\
\hline After & 20 & $2.25 \pm 0.09$ & $87.95 \pm 19.59$ & $52.85 \pm 21.14$ & $16.47 \pm 6.33$ & $57.29 \pm 21.59$ & $63.95 \pm 27.01$ \\
\hline \multicolumn{8}{|l|}{$<50$} \\
\hline Prior to & 25 & $2.27 \pm 0.09$ & $101.32 \pm 52.21$ & $44.86 \pm 16.45$ & $15.93 \pm 6.42$ & $115.16 \pm 78.34$ & $86.48 \pm 41.33$ \\
\hline After & 25 & $2.23 \pm 0.11$ & $85.64 \pm 35.18$ & $47.10 \pm 12.62$ & $15.52 \pm 6.07$ & $78.34 \pm 50.48$ & $71.54 \pm 39.20$ \\
\hline
\end{tabular}

${ }^{\mathrm{a}} \mathrm{P}=0.018(\mathrm{P}<0.05)$. Values are expressed as the mean \pm standard deviation. ALP, alkaline phosphatase; $\mathrm{PTH}$, parathyroid hormone; $25(\mathrm{OH}) \mathrm{D} 3$, 25-hydroxyvitamin D3; P1NP, I collagen N-terminal peptide; CTX, $\beta$-collagen crosslinked C-terminal peptide.

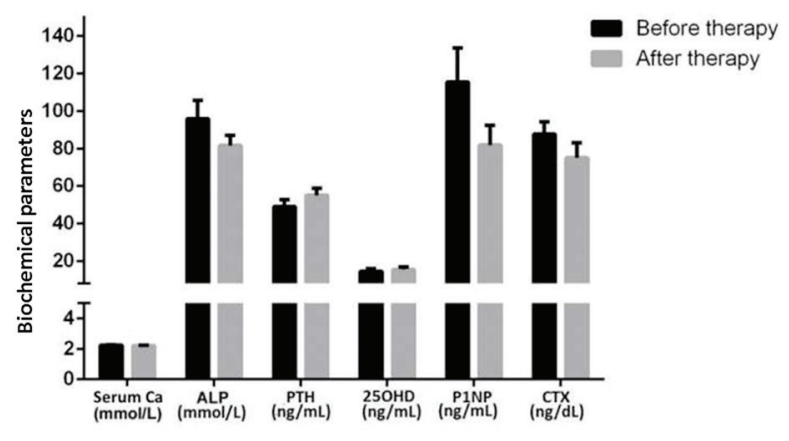

Figure 3. Comparison of serum $\mathrm{Ca}^{2+}, 25(\mathrm{OH}) \mathrm{D} 3, \mathrm{ALP}, \mathrm{PTH}, \mathrm{P} 1 \mathrm{NP}$ and CTX levels in females prior to and after treatment. There was no significant difference in serum Ca, ALP, PTH, 25(OH)D3, P1NP or CTX levels after treatment vs. baseline in females. ALP, alkaline phosphatase; PTH, parathyroid hormone; 25(OH)D3, 25-hydroxyvitamin D3; P1NP, I collagen $\mathrm{N}$-terminal peptide; CTX, $\beta$-collagen crosslinked C-terminal peptide.

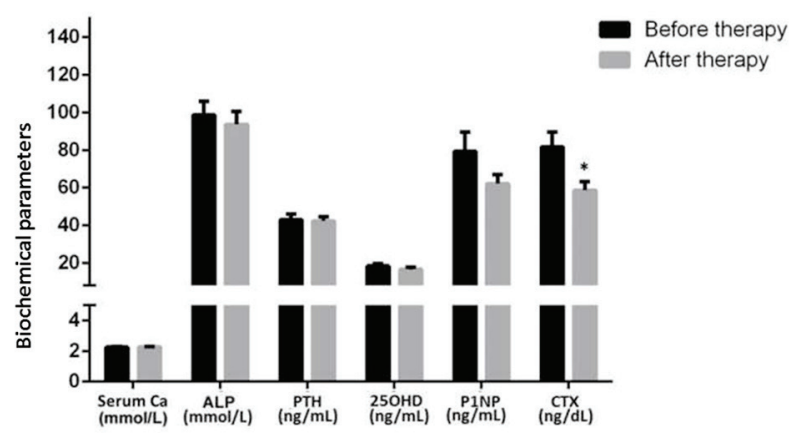

Figure 4. Comparison of serum $\mathrm{Ca}^{2+}$, 25(OH)D3, ALP, PTH, P1NP and CTX levels in males prior to and after treatment. CTX levels were significantly decreased after treatment. ${ }^{\text {}} \mathrm{P}<0.05$ vs. prior to therapy. ALP, alkaline phosphatase; PTH, parathyroid hormone; 25(OH)D3, 25-hydroxyvitamin D3; P1NP, I collagen N-terminal peptide; CTX, $\beta$-collagen crosslinked C-terminal peptide.

Previous studies have confirmed that hyperthyroidism combined with GO affects bone turnover, and that the hip bone density is lower than normal (14). Whether subsequent MPPT further worsens bone metabolism was the focus of the present study.

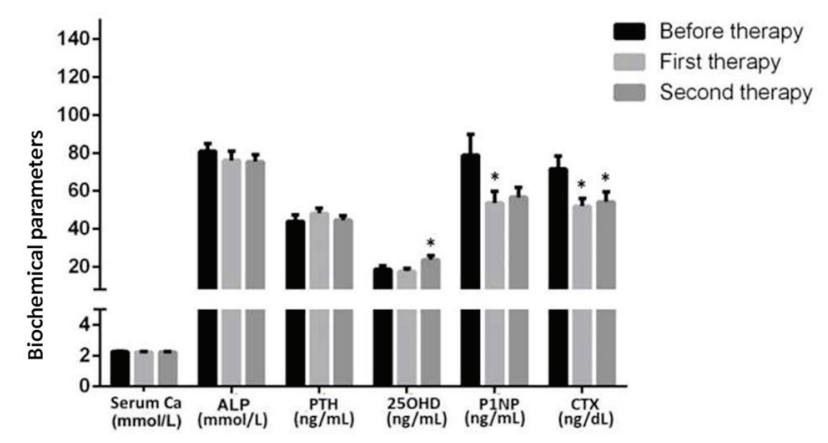

Figure 5. Comparison of serum $\mathrm{Ca}^{2+}, 25(\mathrm{OH}) \mathrm{D} 3, \mathrm{ALP}, \mathrm{PTH}, \mathrm{P} 1 \mathrm{NP}$ and CTX levels in patients subjected to the 2-pulse therapy. After a single pulse, P1NP and CTX levels were significantly lower compared with those at baseline. After the second pulse, CTX was still significantly different vs. that prior to therapy, but there was no significant difference in P1NP. 25(OH)D3 was not significantly altered after the first pulse, but after the second pulse, it was significantly increased compared with that after the single pulse. " $\mathrm{P}<0.05$ as indicated. ALP, alkaline phosphatase; PTH, parathyroid hormone; $25(\mathrm{OH})$ D3, 25-hydroxyvitamin D3; P1NP, I collagen N-terminal peptide; CTX, $\beta$-collagen crosslinked C-terminal peptide.

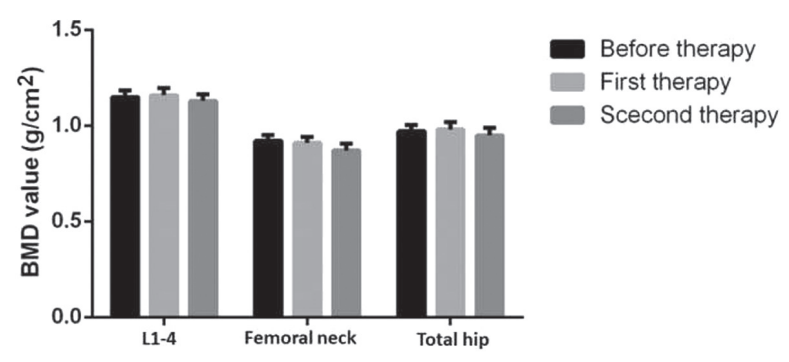

Figure 6. Comparison of BMD $\left(\mathrm{g} / \mathrm{cm}^{2}\right)$ in different anatomic sites of patients who received 2-pulse therapy. There was no significant difference in BMD between the groups. BMD, bone mineral density.

Due to the possible negative effects of hyperthyroidism on bone metabolism, patients whose FT3 and FT4 had been restored to normal were included in the present study; in fact, most patients during treatment at the hospital's outpatient consultations were provided with adjustments to reach the normal range. The present results indicated that after single-pulse treatment, bone formation and bone fracture 
indices of patients were significantly lower than those prior to treatment, Although most studies have revealed that glucocorticoids can lead to increased bone resorption, the results of the current study revealed that the CTX level decreased. This may have been associated with a later period of review, and it is generally believed that early bone resorption increased, but over time, the apoptosis of osteoclasts increased, which is consistent with the previously reported effect of GCs on bone cells in general (12). Whether the latter continuous treatment induces apoptosis of bone cells is certainly worthy of further investigation. In the present study, after the second pulse, the patient's bone transfer marker did not exhibit any further decrease, and no statistically significant differences were observed after the first pulse, suggesting that the 2 pulses did not exert any further inhibitory actions on the skeleton.

The 2016 European Thyroid Association/European Group on Graves' Orbitopathy Guidelines for the Management of Graves' Orbitopathy suggest that intravenous GC pulse treatment should not continue for $>12$ weeks and the cumulative dose of methylprednisolone should not exceed $8 \mathrm{~g}$, thus defining broad boundaries for the safe use of high-dose intravenous GC pulse therapy (9). Although intravenous GC are the first-line effective treatment, partial or inadequate responses or recurrences are not uncommon. It remains a challenge for clinicians to manage these refractory patients. Due to limited evidence, if GO is still active, the above-mentioned guidelines recommend that the cumulative dose of $8 \mathrm{~g}$ of methylprednisolone should not be exceeded in this case. Although there is an increased risk of adverse reactions as the doses increase, adverse effects of GC vary between individuals, and this was not dose-dependent. The adverse reactions mainly comprise metabolic effects and liver lesions, which require careful observation and evaluation by clinicians. After strict monitoring of the patients' tolerance to the drug and treatment response, those patients receiving a second course of therapy were carefully selected, and of note, no further impact on those patients was observed. Other studies also reported on the improved efficacy and safety of high-dose intravenous GC therapy, including liver injury, in the treatment of GO $(15,16)$.

GC are powerful regulators of bone cell growth and differentiation, and their effects on bones and associated tissues depend upon a variety of factors, including dosage, exposure time, type of steroid hormone used and species. Certain effects of GCs in the human body are indirect, including the regulation of intestinal calcium absorption and parathyroid secretion; other effects in the skeletal microenvironment occur as usual cellular responses. The present study reported that the adverse reactions to MPPT were principally focused on the liver, and it is known that age and methylprednisolone dose are important risk factors (17). After the menopause in females, rapid bone loss is observed, and males aged $>50$ years also have a reduction in bone mass. Therefore, patients were stratified according to age, and they were divided into a $\geq 50$-year-old group and a $<50$ year old group. It was observed that CTX levels in individuals aged $\geq 50$ years were lower after treatment $(\mathrm{P}<0.05)$. However, other biochemical markers for bone, including ALP and PTH, were not significantly different after therapy in the 2 groups. Although P1NP was reduced after one course, but no further reduction was exhibited after the second course. A recent study also indicated that post-menopausal females using inhaled GC had substantial abnormalities in Volumetric bone mineral density, microarchitecture and stiffness compared to controls, as determined by high-resolution peripheral computed tomography (18). It is speculated that this effect may be associated with the negative effects of aging on bones and in the present study, males exhibited a significant decline in CTX levels $(\mathrm{P}<0.05)$. Leib and Winzenrieth (19) also reported an influence of sex on TBS, with a decrease of a greater magnitude in men. This is consistent with the present study, which suggested that male bones are more susceptible to GC-induced impairment. During the course of treatment, 16 patients underwent a 2-course pulse regimen, which increased the dosage and duration of GC use, but still exerted no further adverse effects on the bone, including changes in BMD.

The present results indicated that $100 \%$ of patients with hyperthyroidism had vitamin D deficiency, which is consistent with the results of previous studies (14). In patients with vitamin D deficiency or decreased bone mass during methylprednisolone therapy, supplemental calcium and vitamin D is routinely given at our clinic, and patients are encouraged to increase their light exposure and outdoor activities. This may also partly explain the higher levels of vitamin D after a single or double pulse. Similarly, the effectiveness of vitamin D3 (cholecalciferol) in counteracting side effects of GCs has been previously demonstrated $(20,21)$. Certain studies have indicated that prednisolone-induced $\mathrm{GC}$ receptor and receptor activator of NF- $\kappa \mathrm{B}$ ligand (RANKL)/RANK/osteoprotegerin-signaling pathway abnormalities are associated with damage to bone marrow vitamin $\mathrm{D}$ secretion, and it may be improved by supplementation of bile calcification alcohols (22); these may be factors underlying bone protection in the present pulse-therapy protocols.

In summary, the results of the present study confirmed that a 12-week-long pulse therapy for GO (total dose, $4.5 \mathrm{~g}$ ) was safe and effective for the skeleton, and it remained so even with 2 treatments (total dose, $9 \mathrm{~g}$ ). In fact, with the improvement in hyperthyroidism, bone turnover improved, and treatment with another methylprednisolone pulse did not further deteriorate the bone metabolic status-even in adults aged $>50$ years. Similarly, based on the possible adverse effects of GCs on the skeleton, most patients were supplemented with calcium and vitamin D3, which may also have increased bone protection. In terms of the explicit anti-inflammatory and immunologic effects of MPPT therapy, it was demonstrated that short-term, moderate doses of GCs were well-tolerated and exhibited a higher benefit/risk ratio. At the same time, it is recommended to evaluate the risk of fracture and to provide bone protection therapy for such patients, including calcium and vitamin D supplementation.

There are certain weaknesses of the present study, including a limited number of cases, as there were only 16 patients with 2 courses of MPPT treatment. The retrospective nature of the analysis and the lack of a rigorous design regarding the clinical trial process are further limitations. Increased calcium and/or vitamin $\mathrm{D}$ use in the experimental treatments may have also impacted the BMD results. It has been demonstrated that GCs exert adverse effects on vertebral bone microstructure, and they may therefore lead to an increased risk of fracture due to a variety of factors. These include increased risk of falls and changes in bone mass, but these cannot be predicted through 
BMD measurements. In addition, excessive GCs have adverse effects on muscle mass and function, leading to myopathies and an increased risk of fall (23). Evaluation of these aspects will be performed by our group upon later follow-up.

\section{Acknowledgements}

Not applicable.

\section{Funding}

The current study was supported by The Second Batch of Leading Talent of Traditional Chinese Medicine in Jiangsu Province' Project (grant. no. SLJ0209).

\section{Availability of data and materials}

The datasets used and/or analyzed during the current study are available from the corresponding author on reasonable request.

\section{Authors' contributions}

CL, YXH and RDZ conceived and designed the study. YXH, YFF, LS and XH performed the experiments. YXH wrote the paper. YFF, CL and $\mathrm{XH}$ reviewed and edited the manuscript. All authors read and approved the final manuscript.

\section{Ethics approval and consent to participate}

The current study was approved by the Medical Ethics Board of Nanjing University of Chinese Medicine (Nanjing, China). All patients provided written informed consent regarding the use of their clinical data for scientific research.

\section{Patient consent for publication}

Not applicable.

\section{Competing interests}

The authors declare that they have no competing interests.

\section{References}

1. Wiersinga WM and Bartalena L: Epidemiology and prevention of Graves' ophthalmopathy. Thyroid 12: 855-860, 2002.

2. Wiersing WM: Advances in treatment of active, moderateto-severe Graves' ophthalmopathy. Lancet Diabetes Endocrinol 5: 134-142, 2017.

3. Stiebel-Kalish H, Robenshtok E, Hasanreisoglu M, Ezrachi D, Shimon I and Leibovici L: Treatment modalities for Graves ophthalmopathy: Systematic review and meta-analysis. J Clin Endocrinol Metab 94: 2708-2716, 2009.

4. Gao G, Dai J, Qian Y and Ma F: Meta-analysis of methylprednisolone pulse therapy for Graves' ophthalmopathy. Clin Exp Ophthalmol 42: 769-777, 2014
5. Bartalena L, Baldeschi L, Dickinson A, Eckstein A, Kendall-Taylor P, Marcocci C, Mourits M, Perros P, Boboridis K, Boschi A, et al: Consensus statement of the European Group on Graves' Orbitopathy (EUGOGO) on management of GO. Eur J Endocrinol 158: 273-285, 2008.

6. Zang S, Ponto KA and Kahaly GJ: Clinical review: Intravenous glucocorticoids for Graves' orbitopathy: Efficacy and morbidity. J Clin Endocrinol Metab 96: 320-332, 2011.

7. Whittier X and Saag KG: Glucocorticoid-induced Osteoporosis. Rheum Dis Clin North Am 42: 177-168, 2016.

8. Bartley GB and Gorman CA: Diagnostic criteria for Graves' ophthalmopathy. Am J Ophthalmol 119: 792-795, 1995.

9. Bartalena L, Baldeschi L, Boboridis K, Eckstein A, Kahaly GJ, Marcocci C,Perros P,Salvi M,Wiersinga WM,European Group on Graves, et al: The 2016 european Thyroid Association/European Group on Graves' Orbitopathy guidelines for the management of Graves' orbitopathy. Eur Thyroid J 5: 9-26, 2016.

10. Bahn R: High-dose intravenous glucocorticoid therapy for Graves' ophthalmopathy: Where are we now? Thyroid 22: 1-2, 2012.

11. San Miguel I, Arenas M, Carmona R, Rutllan J, Medina-Rivero F and Lara P: Review of the treatment of Graves' ophthalmopathy: The role of the new radiation techniques. Saudi J Ophthalmol 32: 139-145, 2018.

12. Adler RA: Glucocorticoid-induced osteoporosis: Management challenges in older patients. J Clin Densitom 22: 20-24, 2019.

13. Compston J: Glucocorticoid-induced osteoporosis: An update. Endocrine 61: 7-16, 2018.

14. Yongxin H U , Li S , Shaofeng X , et al. Analysis of bone metabolism in patients with Graves' orbitopathy[J]. Jiangsu Medical Journal, 42: 1875-1877, 2016.

15. Uedasakane Y, Kanamoto N, Fushimi Y, Tanaka-Mizuno S, Yasuno S, Miura M, Sone M, Yasoda A, Okada T, Togashi K, et al: Overall safety and efficacy of high-dose and low-dose intravenous glucocorticoid therapy in patients with moderate-to-severe active Graves' ophthalmopathy. Endocr J 63: 703-714, 2016.

16. Liu X, Wang S, Qin L, Qiang W, Dahal M, Fan P, Gao S and Shi B: Short and long-term effects of high-dose intravenous methylprednisolone pulse therapy on thyroid-associated ophthalmopathy. Exp Ther Med 12: 901-908, 2016.

17. Miśkiewicz P, Kryczka A, Ambroziak U, Rutkowska B, Główczyńska R, Opolski G, Kahaly G and Bednarczuk T: Is high dose intravenous methylprednisolone pulse therapy in patients with Graves' orbitopathy safe? Endokrynol Pol 65: 402-413, 2014.

18. Liu Y, Dimango E, Bucovsky M, Agarwal S, Nishiyama K, Guo XE, Shane E and Stein EM: Abnormal microarchitecture and stiffness in postmenopausal women using chronic inhaled glucocorticoids. Osteoporos Int 29: 2121-2127, 2018.

19. Leib ES and Winzenrieth R: Bone status in glucocorticoid-treated men and women. Osteoporos Int 27: 39-48, 2016.

20. Shen L, Ma C, Shuai B and Yang Y: Effects of 1,25-dihydroxyvitamin D3 on the local bone renin-angiotensin system in a murine model of glucocorticoid-induced osteoporosis. Exp Ther Med 13: 3297-3304, 2017

21. Kenanidis E, Potoupnis ME, Kakoulidis P, Leonidou A, Sakellariou GT, Sayegh FE and Tsiridis E: Management of glucocorticoid-induced osteoporosis: Clinical data in relation to disease demographics, bone mineral density and fracture risk. Expert Opin Drug Saf 14: 1035-1053, 2015.

22. Shymanskyi I, Lisakovska O, Mazanova A, Labudzynskyi D and Veliky M: Vitamin D3 modulates impaired crosstalk between RANK and glucocorticoid receptor signaling in bone marrow cells after chronic prednisolone administration. Front Endocrinol (Lausanne) 9: 303, 2018.

23. Sato AY, Richardson D, Cregor M, Davis HM, Au ED, McAndrews K, Zimmers TA, Organ JM, Peacock M, Plotkin LI and Bellido T: Glucocorticoids induce bone and muscle atrophy by tissue-specific mechanisms upstream of E3 ubiquitin ligases. Endocrinology 158: 664-677, 2017. 